downstream effect on adolescents' sense of control over their health and on their risk behaviors. This study aimed to test whether primary care provider (PCP) training on motivational interviewing together with implementation of an electronic screening and feedback tool would impact provider counseling on health risks and adolescent risk behaviors.

Methods We used a stepped wedge study design and recruited adolescents aged 13-18 from 6 clinics. In the control period, 135 youth received their usual primary care appointment. In the intervention period, 167 youth received an electronic screening and feedback tool prior to their visit, with results sent to the PCP. In between the control and intervention periods, PCPs participated in an online interactive training demonstrating motivational interviewing skills using video-based scenarios, and met to discuss clinic-level reports summarizing adolescent-reported receipt of counseling.

Youth were surveyed at baseline and three months about their health risk behaviors, and following the well-child visit regarding the receipt of counseling. We calculated the total number of behaviors counseled on divided by the total number of risk behaviors endorsed and compared across groups using chi-squared analyses. An adjusted linear mixed model was conducted to examine whether the intervention was associated with changes in the mean risk score at the 3month assessment, adjusting for clinic, age, gender, and baseline risk.

Results Control group participants reported receiving counseling on $35 \%$ of moderate risk behaviors compared to $43 \%$ of behaviors for intervention participants $(p=0.009)$. Likewise, adolescents reported receiving counseling on 23\% of high risk behaviors in the control group compared to $36 \%$ of behaviors in the treatment group $(p<0.001)$. The linear mixed model indicted that the intervention was associated with a 0.64 greater reduction in overall risk score at 3month follow up, relative to the control group (95\% CI:$1.03,-0.24 ; p=0.002$ ).

Conclusion Results show promise for electronic screening and feedback and brief provider training to improve the quality and results of healthcare to adolescent patients in primary care settings.

\section{P5 $\quad$ PSYCHOSOMATIC SYMPTOMS OF CHINESE ADOLESCENTS WHO ARE VICTIMS OF BULLYING}

${ }^{1} \mathrm{JM}$ Li, ${ }^{1,2} \mathrm{~T}$ Hesketh*. 'Global Health Centre, Zhejiang University, Hangzhou, China; ${ }^{2}$ Institute for Global Health, University College London, London, UK

\subsection{6/bmjpo-2019-RCPCH-SAHM.13}

Aims The association between bullying and mental health outcomes is well recognized in many countries, but there is little evidence from China. This study aimed to investigate whether there is an association between being bullied and psychosomatic well-being among Chinese middle school children.

Methods This cross-sectional survey study was conducted in 12-15-year-olds in Zhejiang, Henan and Chongqing provinces, representing Eastern Central and Western regions from May to September, 2018. It included two urban and two rural middle schools in each province, so 12 schools in total. Classes were randomly chosen to achieve roughly the same sample size in each year group in each province. A self-completion questionnaire was completed by the students in the classroom setting and included: traditional bullying, cyberbullying, and classic psychosomatic symptoms of headache, abdominal pain and sleep problems. Data analyses were performed with SPSS 24.0.

Results There were 3774 completed questionnaires, and the mean age was 13.58 (SD 0.87). 567(15\%) stated they had experienced only traditional bullying, 520(14\%) only cyberbullying, and $645(17 \%)$ both. The commonest forms of traditional bullying were verbal bullying experienced by 1034 $(27.6 \%)$ and rumour-spreading experienced by $540(14.4 \%)$. Commonest forms of cyberbullying were being teased online, 703(18.6\%) and being excluded online, 690(18.5\%). 491 $(13.3 \%)$ reported they often had headache, $607(16.5 \%)$ abdominal pain, and 597(16.1\%) sleep problems. After adjusting for confounders, we found that traditional-cyber victims (adjusted OR 1.8, 95\% CI 1.4-2.1), only-traditional victims (1.4, 1.1-1.7), only-cyber victims $(1.6,1.3-2.0)$ were more likely to have headache. Traditional-cyber victims (adjusted OR 1.8, 95\% CI 1.5-2.2), only-traditional victims (1.3, 1.0-1.5), only-cyber victims $(1.4,1.1-1.7)$ were more likely to have abdominal pain. Traditional-cyber victims (adjusted OR 2.0, 95\% CI 1.7-2.5), only-cyber victims $(1.4,1.2-1.8)$ were more likely to have sleep problems.

Conclusion The prevalence of bullying victims is substantial among Chinese adolescents, and is associated with psychosomatic conditions. Measures to reduce bullying are needed in Chinese schools. There needs to be increased awareness of the harm caused by bullying.

\section{P6 IMPROVEMENTS IN SOCIAL DETERMINANTS AND DECLINES IN ADOLESCENT PREGNANCY AND CHILD MARRIAGE IN RURAL UGANDA, 1994-2018}

${ }^{1}$ JS Santelli* ${ }^{*}{ }^{2}$ Chen, ${ }^{1} \mathrm{E}$ Spindler, ${ }^{3} \mathrm{~F}$ Nalugoda, ${ }^{4} \mathrm{~L}$ Lindberg, ${ }^{3} \mathrm{~T}$ Lutalo, ${ }^{5} \mathrm{M}$ Wawer, ${ }^{6} \mathrm{~F}$ Ssewamala, ${ }^{7} \mathrm{~S}$ Grilo, ${ }^{8} \mathrm{P}$ Kreniske, ${ }^{8} \mathrm{~S}$ Hoffman, ${ }^{3} \mathrm{~J}$ Kagaayi, ${ }^{3} \mathrm{R}$ Ssekubugu, ${ }^{5} \mathrm{~K}$ Grabowski, ${ }^{5}$ RH Gray. ${ }^{1}$ Population and Family Health, Mailman School of Public Health, Columbia University, New York, USA; ${ }^{2}$ Biostatistics, Mailman School of Public Health, Columbia University, New York, USA; ${ }^{3}$ Rakai Health Sciences Program, Entebbe, Uganda; ${ }^{4}$ Guttmacher Institute, New York, USA; ${ }^{5}$ Department of Epidemiology, Johns Hopkins Bloomberg School of Public Health, Baltimore, USA; ${ }^{6}$ The George Warren Brown School of Social Work, Washington University in St. Louis, St. Louis, USA; ${ }^{7}$ Department of Sociomedical Sciences, Mailman School of Public Health, Columbia University, New York, USA; ${ }^{8}$ HIV Center for Clinical and Behavioral Studies, New York State Psychiatric Institute, New York, USA

\subsection{6/bmjpo-2019-RCPCH-SAHM.14}

Aims To identify the relationships among social determinants, public policies, behaviors, and adolescent pregnancy and child marriage - in the context of a declining HIV epidemic in the Rakai region of rural Uganda. Social determinants may influence key adolescent social transitions such as leaving school, marriage, and childbearing.

Methods Data on young women 15-19 years from 17 surveys (1994-2018) in a population-based, open cohort of households in 28 communities followed continuously since 1994. Social determinants included school enrollment, a household assets measure of socioeconomic status (SES), and orphanhood (death of one or both parents). A previously validated measure (the Pregnancy Risk Index or PRI) was used to estimate young women's risk of becoming 